\title{
Regulating recognition decisions through incremental reinforcement learning
}

\author{
SANGHOON HaN \\ Duke University, Durham, North Carolina \\ AND \\ IAN G. DobBINS \\ Washington University, St. Louis, Missouri
}

\begin{abstract}
Does incremental reinforcement learning influence recognition memory judgments? We examined this question by subtly altering the relative validity or availability of feedback in order to differentially reinforce old or new recognition judgments. Experiment 1 probabilistically and incorrectly indicated that either misses or false alarms were correct in the context of feedback that was otherwise accurate. Experiment 2 selectively withheld feedback for either misses or false alarms in the context of feedback that was otherwise present. Both manipulations caused prominent shifts of recognition memory decision criteria that remained for considerable periods even after feedback had been altogether removed. Overall, these data demonstrate that incremental reinforcement-learning mechanisms influence the degree of caution subjects exercise when evaluating explicit memories.
\end{abstract}

Recognition criteria are hypothetical standards by which memory evidence is categorized as either sufficient or inadequate to warrant a judgment of prior encounter (viz., old) (Macmillan \& Creelman, 1991; see Figure 1). Although most memory researchers assume that criteria are adaptive, there are few models of learning that might support such adaptability (however, see Estes \& Maddox, 1995; Unkelbach, 2006), and to date, the vast majority of successful manipulations of memory decision criteria have involved explicit instructions given to observers about the relative preponderance of old and new items (Hirshman, 1998; Rotello, Macmillan, Reeder, \& Wong, 2005; Strack \& Förster, 1995) or explicit warnings to avoid errors of either omission or commission (Azimian-Faridani \& Wilding, 2006). These instructed criterion shifts are sometimes augmented with clear descriptions of monetary losses and gains attached to different response outcomes (payoff matrices; see Van Zandt, 2000), but in all of these cases, observers consciously attempt to comply with instructions, given their understanding of test list regularities or characteristics. What remains unclear is whether the decision criterion can adapt without an explicit or controlled strategy.

One candidate mechanism we propose that might enable adaptive positioning of a criterion is incremental reinforcement learning, which is central for learning category distinctions in other nonrecognition domains (e.g., Gluck $\&$ Bower, 1988; Poldrack et al., 2005). Such learning requires integrating trial-by-trial feedback outcomes and gradual remapping of different decisions onto different stimulus feature or feature combinations as a function of probabilistic reward likelihood (for a review, see Ashby \& Maddox, 2005). Two category-learning paradigms having this characteristic are information integration and probabilistic classification tasks. During both, the relationship between key stimulus features and appropriate decisions cannot be reduced to a simple explicit, verbalizable strategy, because observers must classify the items on the basis of complex combinations of multiple feature dimensions (e.g., a nonlinear combination of thickness and orientation of sinusoidal gratings) or because feedback is rendered probabilistically, so that making the same judgment for a given repeated stimulus does not guarantee receiving the same feedback outcome on every trial (see also Ashby \& O'Brien, 2007). Neuropsychological findings suggest that learning during these tasks relies heavily upon the integrity of the striatum, a basal ganglia structure linked to implicit procedural and habit learning (Knowlton, Mangels, \& Squire, 1996; Saint-Cyr, Taylor, \& Lang, 1988).

Although feedback-based changes in criteria have frequently been examined in perceptual judgment tasks (e.g., Dorfman \& Biderman, 1971; Thomas, 1973), there are fundamental differences between perceptual classification tasks and the regulation of episodic recognition judgments. More specifically, in feedback-based category-learning tasks, it is assumed that the mapping between object features and category decisions is incrementally altered via trial-by-trial feedback learning. However, during episodic recognition tests, the perceptual and semantic features of the probes are not diagnostic of the required categorical

S.Han, sanghoon.han@duke.edu 


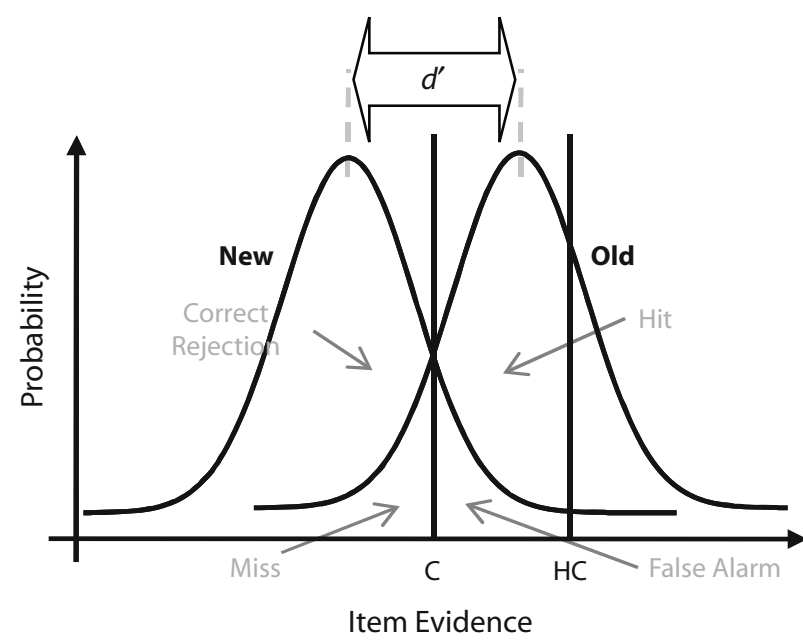

Figure 1. Example of one-dimensional signal detection theory (SDT) model of item-based memory decision. The figure illustrates the model of old/new recognition with normal probability density distributions of familiarity values for old and new items. Accuracy estimate $d^{\prime}$ is the distance between the means of two distributions divided by the standard deviation. " $C$ " denotes the SDT estimate of bias that is the relative position of "old/new" decision criterion with respect to the intersection of two distributions, and "HC" denotes the high confidence criterion. The decision criterion is the hypothetical standard by which memory information is categorized as either sufficient or inadequate to warrant a categorical judgment of old (Macmillan \& Creelman, 1991). Old items whose evidence falls above the criterion are correctly endorsed (hits), whereas new items with evidence above the criterion yield errors of commission (false alarms). The complementary proportions below the criterion are labeled "correct rejection" and "miss," respectively.

distinction, since the memory status of the probes and the types of features each possesses are orthogonal. Instead, incremental reinforcement learning, if successful, must alter the mapping between levels of retrieved memory evidence and recognition decisions, and this represents a level of abstraction not found during perceptual classification learning. In addition, observers cannot learn a reinforced response to each individual test item, because the items within a memory test are never repeated.

Perhaps consistent with the notable differences between episodic recognition and perceptual classification demands, evidence for the efficacy of feedback regulation of recognition criteria has been decidedly mixed. For example, studies by Estes and Maddox (1995) and Healy and Kubovy (1977) failed to demonstrate criterion shifts during recognition paradigms that manipulated the base rates of studied items in conjunction with trial-based feedback procedures, although similar procedures easily produced shifts in their perceptual classification tasks. In contrast, Rhodes and Jacoby (2007) manipulated the relative item probabilities across two screen locations and found that, in conjunction with trial-based feedback, subjects demonstrated different recognition criteria for the two locations. However, the criterion shifts were prominent only for observers explicitly aware of the location manipulation of target density, and the removal of the feedback greatly reduced the criterion difference. Criti- cally, neither explicit awareness nor continued presence of feedback reinforcement should have been necessary if incremental reinforcement learning had governed the effects. Using a different procedure, Verde and Rotello (2007) demonstrated an acquired criterion shift for separate halves of a test list containing well-encoded versus poorly encoded study items intermixed with lures. This design also used trial-based feedback, in addition to halting the test and providing performance summaries during testing. Although these researchers did not examine observer awareness of the test list characteristics, it is possible that the feedback and, particularly, the performance summaries may have explicitly alerted the subjects to the fact that the well-encoded and poorly encoded study items were not distributed evenly across test halves. In total, although these designs importantly demonstrated criterion flexibility during the course of testing, they point toward a mechanism based on explicit awareness of test list regularities. In addition, they confound a manipulation of the test list characteristics with the presence of trial-based feedback, which necessarily precludes assigning an exclusive role to the processing of feedback in the observed criterion shifts.

Despite the limited support for an incremental reinforcement-learning mechanism governing episodic recognition, recognition decisions arguably share important similarities to feedback-based classification-learning tasks. First, episodic information is often assumed to be multidimensional (Johnson, Hashtroudi, \& Lindsay, 1993; Yonelinas, 1994), so that the category old may depend on complex combinations of different trace attributes in ways difficult to capture in a simple explicit response strategy. Second, under most measurement models of recognition, any decision criterion for responding will yield positive feedback only probabilistically, because the evidence evoked by old and new items overlaps and cannot be fully separated by a simple criterion boundary (Macmillan \& Creelman, 1991; see Figure 1). These two characteristics suggest that recognition judgments may be influenced by the same learning mechanisms shown to govern classification learning in information integration and/or probabilistic classification tasks, provided such mechanisms are sensitive to abstract mnemonic evidence representations.

One study suggesting such a mechanism underlying learned criterion shifts is Han and Dobbins (2008), in which systematically misleading feedback was used to shift the relative criteria of two recognition groups. During the procedure, one group was given false positive feedback for errors of commission (false alarms), and the other was given false positive feedback for errors of omission (misses). All other feedback was correct. This design isolated any criterion shift solely to the nature of the feedback, since the actual structure of the test lists remained equivalent across the groups. The manipulation shifted the relative criteria of the groups, and this difference remained even when feedback became fully correct in the second test block of the design, suggesting a durable form of learning (cf. Rhodes \& Jacoby, 2007). In addition, the majority of subjects did not report any perceived anomalies in the feedback itself, posttest. 
Although it was suggestive of incremental reinforcement learning, there were potential drawbacks to the design in Han and Dobbins (2008). First, the feedback was fully deterministic, in that every error of a particular kind received the false positive feedback. For example, in the condition designed to instill a lax criterion, all false alarms were incorrectly cued as correct responses. This meant that no old response ever received a negative feedback outcome for this group. Such deterministic feedback procedures are known to shift learning toward explicit rule use and away from incremental reinforcement learning, with probabilistic versus deterministic feedback conditions potentially engaging different neural learning systems (e.g., Frank \& Kong, 2008; Mehta \& Williams, 2002; Robinson, Heaton, Lehman, \& Stilson, 1980). Second, the design relied exclusively upon false positive feedback in order to shift the criteria. This approach was chosen because it was assumed that subjects would be uncertain during the commission of errors and, hence, the manipulation of the feedback validity would be difficult to detect. Nonetheless, this also potentially weds the manipulation exclusively to surprising event outcomes. Although the reinforcement literature suggests that this may be particularly useful for learning, since it should evoke considerable "positive prediction error" (Schultz, 2000), it may also increase the likelihood of explicit awareness of the manipulation. Finally, the design of Han and Dobbins failed to demonstrate that the criterion shifts survived complete removal of the feedback. Since a hallmark of successful incremental reinforcement learning is the perseverance of decision tendencies in the absence of any form of external reinforcement (e.g., Cincotta \& Seger, 2007), it is critical to demonstrate that the acquired memory criterion shifts remain for some notable period, absent feedback. Thus, the goal of the present study was to examine whether feedback-based memory criterion shifts would demonstrate three key properties consistent with incremental reinforcement-learning processes - namely, that they should be (1) sensitive to probabilistic feedback contingencies, (2) not solely dependent on surprising false positive outcomes, and (3) persistent in the complete absence of supporting feedback.

\section{GENERAL METHOD}

\section{Subjects}

Sixty-four Duke undergraduates (30 in Experiment 1, 34 in Experiment 2) participated in return for partial course credit. Informed consent was obtained as required by the human subjects review committee of Duke University. In Experiment 1, a postexperiment questionnaire was administered asking about the feedback procedures, to assess participant awareness of the manipulation. One subject who correctly believed the feedback to be inaccurate or skewed was removed from Experiment 1.

\section{Materials and Procedure}

In Experiments 1 and 2, four lists of 200 word items (average of 7.09 letters and 2.34 syllables, with a Kučera-Francis corpus frequency of $8.85 ; 100$ studied and 100 lure items for each cycle) were constructed for use in sequential study/test cycles. List and condition assignment was randomized for each subject. During study, the subjects rated words on the computer screen for the number of syllables ("Counting syllables $1 / 2 / 3$ /more than 4 ") within a limited amount of time ( $2 \mathrm{sec}$ ), immediately followed by a forewarned memory test. The subjects were not forewarned that feedback would be present during testing. In each test, studied and lure items were randomly intermixed and presented serially for self-paced old/new recognition judgments. Following the old/new response, the subjects rated confidence on a scale of $1-3$ ("Confidence? Unsure $=123=$ Certain"). Feedback, when given, immediately followed the confidence report. The key and only manipulation across experiments was the nature of the feedback.

Probabilistic biased feedback manipulation. In Experiment 1 , the validity of the feedback given to errors was probabilistically altered in order to tacitly encourage lax or strict responding. More specifically, a random portion of a particular type of error (misses or false alarms) was incorrectly reported as "correct." The subjects were correctly informed during correct responses (hits and correct rejections). Consistent with incremental reinforcement-learning principles, the general expectation was that the subjects would learn to favor the decision more often linked to a positive feedback outcome ("correct" feedback indication) and/or would learn to avoid the response option that more often led to a negative outcome ("incorrect" feedback indication). The false-feedback manipulation was restricted to errors, since they are typically of low confidence; hence, incorrect feedback should not have raised suspicions on the part of the subjects. In Experiment 2, the balance of positive/negative feedback was instead shifted by simply omitting correct negative feedback for one or the other class of error (availability manipulation). The analyses employed the detection theoretic estimate of accuracy, $A_{z}$ (Rotello, Masson, \& Verde, 2008), and a criterion, $c$.

\section{EXPERIMENT 1 Criterion Learning Based on Probabilistic False Feedback}

The goal of Experiment 1 was to determine whether a probabilistic variant of the false-feedback procedure would induce criterion shifts. Half of the subjects were given false positive feedback "That is CORRECT" for approximately $70 \%$ of their incorrect old classifications of new items (false alarms). All other responses received correct feedback. We will refer to this as the lax condition (L). For the other half of the subjects, approximately $70 \%$ of incorrect new classifications of old items (misses) received false positive feedback (strict condition [S]). Each group received the same manipulation ( $\mathrm{L}$ or $\mathrm{S}$ ) on the first two successive study/test cycles. Following this, two additional study/test cycles were given with no feedback whatsoever during testing (no feedback $[\mathrm{N}]$ ). This allowed us to assess the durability of criterion learning in the absence of any external reinforcement. Thus there were two groups, one receiving LLNN feedback conditions and the other SSNN.

\section{Results and Discussion}

$\operatorname{Accuracy}\left(\boldsymbol{A}_{z}\right)$. A two-way ANOVA for $A_{z}$, with factors of group (LLNN or SSNN) and test (first, second, third, or fourth), yielded no main effect of group $(p>.84)$ or test $(p>.09)$ and no evidence for an interaction between group and test $(p>.32)$, suggesting that the groups displayed similar accuracy during each test (Table 1).

Decision criterion (c). An ANOVA for decision criterion $c$, with factors of group and test, revealed a main effect of group $\left[F(1,28)=11.85, p<.01, \eta_{\mathrm{p}}^{2}=.30\right]$, with the SSNN group demonstrating a more conserva- 
Table 1

Experiment 1: Accuracy and Decision Criterion Estimates Across Groups and Tests

\begin{tabular}{|c|c|c|c|c|c|c|c|c|c|c|c|c|c|c|c|c|}
\hline & \multicolumn{16}{|c|}{ Group } \\
\hline & \multicolumn{8}{|c|}{ Strict-Strict-No-FB-No-FB } & \multicolumn{8}{|c|}{ Lax-Lax-No-FB-No-FB } \\
\hline & \multicolumn{2}{|c|}{ Test 1} & \multicolumn{2}{|c|}{ Test 2} & \multicolumn{2}{|c|}{ Test 3} & \multicolumn{2}{|c|}{ Test 4} & \multicolumn{2}{|c|}{ Test 1} & \multicolumn{2}{|c|}{ Test 2} & \multicolumn{2}{|c|}{ Test 3} & \multicolumn{2}{|c|}{ Test 4} \\
\hline & $M$ & $S D$ & $M$ & $S D$ & $M$ & $\overline{S D}$ & $M$ & $\overline{S D}$ & $M$ & $S D$ & $M$ & $S D$ & $M$ & $\overline{S D}$ & $M$ & $S D$ \\
\hline Hit & .71 & .10 & .68 & .13 & .67 & .17 & .63 & .17 & .76 & .07 & .78 & .07 & .78 & .08 & .74 & .08 \\
\hline False alarm & .15 & .08 & .17 & .08 & .20 & .11 & .20 & .09 & .20 & .07 & .26 & .10 & .31 & .17 & .29 & .13 \\
\hline$A_{z}$ & .83 & .10 & .83 & .06 & .81 & .10 & .79 & .08 & .85 & .04 & .81 & .08 & .79 & .10 & .82 & .08 \\
\hline $\begin{array}{l}\text { Percentage of } \\
\text { manipulated trials }\end{array}$ & 20.50 & 6.76 & 21.30 & 8.16 & & & & & 14.80 & 4.23 & 18.90 & 6.76 & $\mathrm{~N}$ & & & \\
\hline
\end{tabular}

Note-FB, feedback.

tive criterion (mean $c=.24$ ) than did the LLNN group $(-.03)$. There was no main effect of test $(p>.19)$ and no evidence for an interaction between group and test $(p>$ $.65)$, suggesting a persistent difference in criterion across the two groups regardless of test. Pairwise comparisons of the groups' criteria during each of the four separate tests were all significant $[t(28)=2.05,3.37,2.57$, and 2.48 , respectively], although the smallest numerical difference in criteria across the groups was during Test 1 (see Table 1).

The probabilistic nature of the present feedback manipulation would have precluded the belief that a given type of response never resulted in errors, yet a relative shift was nonetheless induced. Furthermore, the no-feedback condition ruled out interpretations that necessarily rely on the continued presence of feedback. For example, if the criterion shift represented a trial-to-trial win-stay strategy (Frank \& Kong, 2008) on the part of the subjects, removing feedback should have eliminated the relative criterion differences. Finally, we parsed Tests 1 and 2 into subblocks (cumulative blocks of 40 trials $[40,80,120,160$, and 200 trials]) to examine the emergence of the relative shift of criterion $c$ in a finer grained manner within each test. Test 1 yielded a significant interaction $[F(4,92)=2.86$, $\left.p<.05, \eta_{\mathrm{p}}^{2}=.11\right]$ between group and cumulative subblock, reflecting an increasingly larger criterion group difference as the total amount of false feedback accumulated within the test. The same analysis during Test 2 yielded a main effect of group $\left[F(1,23)=7.52, p<.05, \eta_{\mathrm{p}}^{2}=.25\right]$ and no evidence for the interaction between group and subblock ( $p=.82)$, suggesting that the relative difference acquired during Test 1 had already reached asymptote and was carried largely intact into Test 2 . Partially supporting this conclusion, when the criterion measures for each group were compared across the tests, the SSNN group showed no difference between Tests 1 and $2(p>.96)$, although the LLNN group did show a more liberal criterion in Test 2 versus Test $1[t(14)=2.48, p<.05]$. Overall, these findings suggest that some continued learning may have taken place across Tests 1 and 2, but that the vast majority of criterion learning occurred prior to the conclusion of Test 1 , as indicated by a failure to find a group $\times$ cumulative subblock interaction in the second test. These findings are consistent with an incrementally learned recognition decision tendency. ${ }^{1}$

\section{EXPERIMENT 2 \\ Criterion Learning Based on Net Feedback Outcomes}

On the basis of the prior findings, it could be argued that it was the unexpectedly positive outcomes of the manipulated feedback trials that were particularly important for the learning (e.g., Butterfield \& Metcalfe, 2001; Schmidt, Young, Swinnen, \& Shapiro, 1989). Although this would not preclude a core role for incremental reinforcement learning, it should nonetheless be possible to demonstrate adaptive criteria whenever the balance of positive to negative reinforcement systematically favors one decision. Experiment 2 differentially reinforced the judgments by withholding feedback for certain types of errors. Thus, from the subject's perspective, some small portion of trials simply failed to elicit feedback. These neutral, uninformative feedback trials should not reflect unexpectedly positive (or negative) outcomes, but they nonetheless would serve to shift the balance of reinforcement for the two decision types. For half of the subjects, the first two tests selectively encouraged lax responding by eliminating the negative feedback for their false alarms. All other response types were correctly identified by the feedback (lax condition). For the other half of the subjects, their miss responses received no feedback (strict condition). Thus, for each group, one response type was associated with positive and negative outcomes, whereas the other was associated with positive and neutral (nofeedback) outcomes. Again, all feedback was eliminated during Tests 3 and 4 (LLNN or SSNN).

\section{Results and Discussion}

Accuracy. An ANOVA for $A_{z}$, with factors of group and test, yielded a significant main effect only of test $\left[F(3,96)=6.29, p<.001, \eta_{\mathrm{p}}^{2}=.16\right]$, with accuracy gradually declining across the entire experiment. Importantly, there was no interaction between group and test $(p>.30$; see Table 2).

Decision criteria. An ANOVA for $c$, with factors of group and test, revealed a main effect of group $[F(1,32)=$ $11.20, p<.01, \eta_{\mathrm{p}}^{2}=.25 ; .34$ vs. -.05 for the $\mathrm{SSNN}$ vs. the LLNN group, respectively]. There was no main effect of test $(p>.11)$ and no interaction between group and test $(p>.07)$. All pairwise comparisons of the groups' 
Table 2

Experiment 2: Accuracy and Decision Criterion Estimates Across Groups and Tests

\begin{tabular}{|c|c|c|c|c|c|c|c|c|c|c|c|c|c|c|c|c|}
\hline & \multicolumn{16}{|c|}{ Group } \\
\hline & \multicolumn{8}{|c|}{ Strict-Strict-No-FB-No-FB } & \multicolumn{8}{|c|}{ Lax-Lax-No-FB-No-FB } \\
\hline & \multicolumn{2}{|c|}{ Test 1} & \multicolumn{2}{|c|}{ Test 2} & \multicolumn{2}{|c|}{ Test 3} & \multicolumn{2}{|c|}{ Test 4} & \multicolumn{2}{|c|}{ Test 1} & \multicolumn{2}{|c|}{ Test 2} & \multicolumn{2}{|c|}{ Test 3} & \multicolumn{2}{|c|}{ Test 4} \\
\hline & $M$ & $S D$ & $M$ & $S D$ & $M$ & $S D$ & $M$ & $S D$ & $M$ & $S D$ & $M$ & $S D$ & $M$ & $S D$ & $M$ & $S D$ \\
\hline Hit & .63 & .17 & .59 & .17 & .59 & .15 & .59 & .16 & .73 & .10 & .74 & .13 & .77 & .10 & .68 & .12 \\
\hline False alarm & .16 & .09 & .16 & .09 & .21 & .11 & .22 & .13 & .26 & .13 & .33 & .16 & .34 & .19 & .33 & .16 \\
\hline$A_{z}$ & .78 & .11 & .77 & .09 & .75 & .07 & .74 & .09 & .82 & .06 & .77 & .07 & .79 & .08 & .74 & .08 \\
\hline$c$ & .33 & .35 & .40 & .38 & .31 & .39 & .31 & .41 & .04 & .33 & -.09 & .40 & -.15 & .38 & .00 & .36 \\
\hline $\begin{array}{l}\text { Percentage of } \\
\text { manipulated trials }\end{array}$ & 36.80 & 16.67 & 40.50 & 17.08 & & & & & 25.60 & 12.88 & 33.00 & 16.21 & $\mathrm{~N} /$ & & & \\
\hline
\end{tabular}

criteria at each of the four separate tests were significant $[t(32)=2.48,3.61,3.50$, and 2.43 , respectively], although again, the smallest numerical difference in criteria across the groups was during the first test (Table 2). To our knowledge, this is the first demonstration that the selective availability of feedback can be used to guide memory decision criterion placement, or criterion placement in general. Again, a finer grained analysis by cumulative test subblocks revealed a clear interaction within Test 1 between group and subblock $[F(4,112)=10.98$, $\left.p<.01, \eta_{\mathrm{p}}^{2}=.28\right]$, suggesting a gradual acquisition of the learned criterion as withheld feedback accrued. The same analysis during Test 2 merely approached significance $\left[F(4,116)=2.40, p=.053, \eta_{\mathrm{p}}^{2}=.08\right]$, suggesting a small increase in criterion differences as further withheld feedback accrued. When Tests 1 and 2 criterion measures were directly compared for each group, the differences were not significant across the tests for either the SSNN group $(p>.26)$ or the LLNN group $(p>.09)$. As in Experiment 1 , this overall pattern suggests that the bulk of criterion learning occurred during the initial test, although some small degree of additional learning or relearning may have occurred during the second test.

\section{GENERAL DISCUSSION}

A fundamental role for incremental reinforcement learning in episodic memory judgments has not been suggested in humans (cf. Wixted \& Gaitan, 2002, for nonhuman animals). Although it is difficult, if not impossible, to completely rule out a role for explicitly maintained strategies in criterion shift experiments (Unkelbach, 2006), and using awareness questionnaires potentially taps only a portion of subject awareness (e.g., Merikle \& Reingold, 1991), it is noteworthy that none of the subjects included here reported awareness of the biased nature of the feedback manipulations. Furthermore, the present findings are quite similar to other classification-learning phenomena that do not require explicit awareness of reward contingencies for learning. In total, these considerations support the notion that the present effects do not require subjects to formulate explicit rule-based strategies in reaction to the biased feedback manipulations, and they clearly demonstrate that no alteration in the test materials themselves is necessary in order to induce a criterion shift (cf. Rhodes
\& Jacoby, 2007; Verde \& Rotello, 2007). Instead, an incremental reinforcement learning framework suggests that the present manipulations led to shifted decision preferences based on the relation of positive/negative outcomes and levels of recognition evidence.

The present data add to early evidence suggesting different routes to regulating episodic recognition decisions. The first, which has been extensively documented in the recognition literature, is an explicit strategy on the part of observers, typically formed following overt warnings or instructions. Furthermore, in those cases in which feedback accompanied a detectable criterion shift for altered lists (e.g., Rhodes \& Jacoby, 2007), the feedback likely alerted the subjects to the list manipulation and, thus, likely represents a strategy similar to those adopted by subjects following explicit instructions or warnings about the riskiness of certain responses. In contrast, the present findings suggest that subjects also appear to develop, through reinforcement learning, incrementally acquired tendencies that durably change the mapping of memory evidence types or levels onto decisions. Similar to the acquisition habits in other domains, these learned criterion shifts may not require subjects to maintain the intention of responding liberally or conservatively across the multiple trials of the test. Because current models of episodic recognition judgment typically do not assume two independent or partially independent decision influences, future work directly contrasting and attempting to doubly dissociate these putative mechanisms, using various methods (i.e., behavioral, functional neuroimaging, special populations), holds promise for further elucidating the mechanisms that regulate the translation of memory content into judgments.

\section{AUTHOR NOTE}

Correspondence concerning this article should be addressed to S. Han, Center for Cognitive Neuroscience, Duke University, Durham, NC 27708 (e-mail: sanghoon.han@duke.edu).

\section{REFERENCES}

Ashby, F. G., \& Maddox, W. T. (2005). Human category learning. Annual Review of Psychology, 56, 149-178.

Ashby, F. G., \& O'Brien, J. B. (2007). The effects of positive versus negative feedback on information-integration category learning. Perception \& Psychophysics, 69, 865-878.

AZIMIAN-FARIDANI, N., \& Wilding, E. L. (2006). The influence of cri- 
terion shifts on electrophysiological correlates of recognition memory. Journal of Cognitive Neuroscience, 18, 1075-1086.

Butterfield, B., \& Metcalfe, J. (2001). Errors committed with high confidence are hypercorrected. Journal of Experimental Psychology: Learning, Memory, \& Cognition, 27, 1491-1494.

Cincotta, C. M., \& Seger, C. A. (2007). Dissociation between striatal regions while learning to categorize via feedback and via observation. Journal of Cognitive Neuroscience, 19, 249-265.

Dorfman, D. D., \& Biderman, M. (1971). A learning model for a continuum of sensory states. Journal of Mathematical Psychology, $\mathbf{8}$, 264-284.

Estes, W. K., \& MADDOX, W. T. (1995). Interactions of stimulus attributes, base rates, and feedback in recognition. Journal of Experimental Psychology: Learning, Memory, \& Cognition, 21, 1075-1095.

Frank, M. J., \& Kong, L. (2008). Learning to avoid in older age. Psychology \& Aging, 23, 392-398.

GLUCK, M. A., \& Bower, G. H. (1988). Evaluating an adaptive network model of human learning. Journal of Memory \& Language, 27, 166195.

Han, S., \& Dobbins, I. G. (2008). Examining recognition criterion rigidity during testing using a biased-feedback technique: Evidence for adaptive criterion learning. Memory \& Cognition, 36, 703-715.

Healy, A. F., \& Kubovy, M. (1977). A comparison of recognition memory to numerical decision: How prior probabilities affect cutoff location. Memory \& Cognition, 5, 3-9.

Hirshman, E. (1998). On the utility of the signal detection model of the remember-know paradigm. Consciousness \& Cognition, 7, 103-107.

Johnson, M. K., Hashtroudi, S., \& Lindsay, D. S. (1993). Source monitoring. Psychological Bulletin, 114, 3-28.

Knowlton, B. J., Mangels, J. A., \& SQuire, L. R. (1996). A neostriatal habit learning system in humans. Science, 273, 1399-1402.

Macmillan, N. A., \& Creelman, C. D. (1991). Detection theory: A user's guide. New York: Cambridge University Press.

Menta, R., \& Williams, D. A. (2002). Elemental and configural processing of novel cues in deterministic and probabilistic tasks. Learning \& Motivation, 33, 456-484.

MerikLe, P. M., \& Reingold, E. M. (1991). Comparing direct (explicit) and indirect (implicit) measures to study unconscious memory. Journal of Experimental Psychology: Learning, Memory, \& Cognition, 17, 224-233.

Poldrack, R. A., Sabb, F. W., Foerde, K., Tom, S. M., Asarnow, R. F., BOOKHEIMER, S. Y., \& KNOWLTON, B. J. (2005). The neural correlates of motor skill automaticity. Journal of Neuroscience, 25, 5356-5364.

Rhodes, M. G., \& JACOBY, L. L. (2007). On the dynamic nature of response criterion in recognition memory: Effects of base rate, awareness, and feedback. Journal of Experimental Psychology: Learning, Memory, \& Cognition, 33, 305-320.

Robinson, A. L., Heaton, R. K., Lehman, R. A. W., \& Stilson, D. W. (1980). The utility of the Wisconsin Card Sorting Test in detecting and localizing frontal lobe lesions. Journal of Consulting \& Clinical Psychology, 48, 605-614.

Rotello, C. M., Macmillan, N. A., Reeder, J. A., \& Wong, M. (2005). The remember response: Subject to bias, graded, and not a process-pure indicator of recollection. Psychonomic Bulletin \& Review, 12, 865-873.

Rotello, C. M., Masson, M. E. J., \& Verde, M. F. (2008). Type I error rates and power analyses for single-point sensitivity measures. Perception \& Psychophysics, 70, 389-401.

Saint-Cyr, J. A., Taylor, A. E., \& Lang, A. E. (1988). Procedural learning and neostriatal dysfunction in man. Brain, 111, 941-959.

Schmidt, R. A., Young, D. E., Swinnen, S., \& Shapiro, D. C. (1989). Summary knowledge of results for skill acquisition: Support for the guidance hypothesis. Journal of Experimental Psychology: Learning, Memory, \& Cognition, 15, 352-359.

Schultz, W. (2000). Multiple reward signals in the brain. Nature Reviews Neuroscience, 1, 199-207.

STRACK, F., \& FörSTER, J. (1995). Reporting recollective experiences: Direct access to memory systems? Psychological Science, 6, 352358.

Thomas, E. A. (1973). On a class of additive learning models: Errorcorrecting and probability matching. Journal of Mathematical Psychology, 10, 241-264.

UnKelbach, C. (2006). The learned interpretation of cognitive fluency. Psychological Science, 17, 339-345.

VAN ZANDT, T. (2000). ROC curves and confidence judgments in recognition memory. Journal of Experimental Psychology: Learning, Memory, \& Cognition, 26, 582-600.

Verde, M. F., \& Rotello, C. M. (2007). Memory strength and the decision process in recognition memory. Memory \& Cognition, 35, 254-262.

Wixted, J. T., \& Gaitan, S. C. (2002). Cognitive theories as reinforcement history surrogates: The case of likelihood ratio models of human recognition memory. Animal Learning \& Behavior, 30, 289-305.

Yonelinas, A. P. (1994). Receiver-operating characteristics in recognition memory: Evidence for a dual-process model. Journal of Experimental Psychology: Learning, Memory, \& Cognition, 20, 13411354.

\section{NOTE}

1. Although we do not present the data, due to space considerations, individual variability in the number of false-feedback trials modulated the size of induced criterion shifts. For example, a median split of observers receiving high versus low amounts of manipulated feedback demonstrated that the criterion shift was more prominent for subjects receiving high amounts of manipulated feedback during Experiments 1 and 2. It was not significant when the low subgroups were compared at each test level. Of course, this outcome would be expected if the feedback manipulation were the cause of the shift, and variability in the composition of feedback across subjects is inherent in all designs that use individual performance feedback to modulate behavior (e.g., Rhodes \& Jacoby, 2007).

(Manuscript received September 13, 2008; revision accepted for publication February 10, 2009.) 